\title{
GENERATION AND INITIAL VALIDATION OF A NEW CASMO5 ENDF/B-VIII.0 NUCLEAR DATA LIBRARY
}

\author{
Rodolfo Ferrer and Joel Rhodes
}

\author{
Studsvik Scandpower \\ 1070 Riverwalk Dr., Suite 150 \\ Idaho Falls, ID 83402 \\ rodolfo.ferrer@studsvik.com, joel.rhodes@studsvik.com
}

\begin{abstract}
A new nuclear data library for the CASMO5 advanced lattice physics code has been generated based on the recently-released ENDF/B-VIII.0 evaluation. The ENDF/B-VIII.0 evaluation represents the state-of-the-art in nuclear data and features new evaluations from the CIELO project for ${ }^{1} \mathrm{H},{ }^{16} \mathrm{O},{ }^{56} \mathrm{Fe},{ }^{235} \mathrm{U},{ }^{238} \mathrm{U}$ and ${ }^{239} \mathrm{Pu}$. A description of the library generation procedure used to process these data into the CASMO5 586 energy group structure is provided. Initial validation of the new ENDF/B-VIII.0-based library, referred to as the E8R0 library, is also presented and involves the comparison of predicted $\mathrm{k}$-eff and fission rate distributions to measurements from various critical experiments. The critical experiments used in the initial validation of the E8R0 library consist of the B\&W 1810 series, B\&W 1484 series, DIMPLE S06A/B, and TCA reflector experiment with iron plates. The results from the initial validation indicate that the new E8R0 library provides a satisfactory performance in terms of CASMO5 predicted k-eff and fission distributions.
\end{abstract}

KEYWORDS: CASMO, ENDF/B-VIII.0, lattice physics, critical experiments

\section{INTRODUCTION}

The fundamental nuclear data used in the generation of multi-group, incident-neutron cross sections is the starting point and foundation of all nuclear reactor simulation and analysis. Advanced in-core fuel management tools, such as Studsvik's Core Management System 5 (CMS5), rely on the two-step scheme for production-level efficient design, analysis and optimization of Light Water Reactors (LWRs). The CMS5 two-step scheme involves the use of the CASMO5 [1] advanced lattice physics code for the generation of homogenized data for downstream use by the SIMULATE5 [2] advanced three-dimensional nodal simulator, which supports the analysis of Boiling Water Reactors (BWRs), Pressurized Water Reactors (PWRs) [3] and VVERs [4,5].

The release of a new nuclear data evaluation affords the opportunity to use the best-available data to possibly reduce biases and improve predictions in LWR analysis. However, experience indicates that careful testing and validation are necessary to determine whether the new and updated data may be regarded as an improvement. Furthermore, the processing of the basic nuclear data evaluation may require changes to the library generation procedure. 
The objectives of this work are to provide a description of the library generation procedure used to process the recently-released ENDF/B-VIII.0 [6] evaluation into a new commercially available CASMO5 586 energy-group library, referred to henceforth as the E8R0 library, and present the initial validation of the E8R0 library using several critical experiments.

\section{ENDF/B-VIII.0 NUCLEAR DATA EVALUATION}

The ENDF/B-VIII.0 nuclear data evaluation was released by the Cross Section Evaluation Working Group (CSEWG) on February 2, 2018. ENDF/B-VIII.0 represents the state-of-the-art in nuclear data, and the neutron sub-library features the following highlights:

- New CIELO evaluations for ${ }^{1} \mathrm{H},{ }^{16} \mathrm{O},{ }^{56} \mathrm{Fe},{ }^{235} \mathrm{U},{ }^{238} \mathrm{U}$ and ${ }^{239} \mathrm{Pu}$, including prompt fission spectra.

- New evaluated data for light nuclides such as ${ }^{3} \mathrm{He},{ }^{6} \mathrm{Li},{ }^{10} \mathrm{~B},{ }^{12} \mathrm{C}$ and ${ }^{13} \mathrm{C}$.

- New evaluated data for structural materials such as ${ }^{54,56-58} \mathrm{Fe},{ }^{58-62,64} \mathrm{Ni},{ }^{59} \mathrm{Co},{ }^{63,65} \mathrm{Cu},{ }^{174-182} \mathrm{Hf}$, and ${ }^{182-186} \mathrm{~W}$.

- Updated evaluated data for minor actinide nuclides such as ${ }^{236 \mathrm{~m}} \mathrm{~Np},{ }^{240} \mathrm{Pu}$, and ${ }^{241,243} \mathrm{Am}$.

- Revised or reevaluated thermal scattering law for $\mathrm{UO}_{2}$, light water, and graphite.

- New data for fission energy release and radioactive decay data.

All major nuclides present in current-generation LWR fuel, coolant, absorber and structural materials possess new evaluations in the ENDF/B-VIII.0 release. Consequently, this new evaluation may impact the prediction of core parameters such as k-eff, temperature coefficients, cycle length, reflector savings, activation and spent fuel characterization. Although the delayed neutron data remains relatively unchanged from the previous release, reactor kinetics predictions may also be indirectly impacted by the new evaluation, primarily due to changes in the fuel temperature (Doppler) coefficient [7]. A detailed description of the ENDF/B-VIII.0 library may be found in reference [6].

Although the total number of nuclides available in the ENDF/B-VIII.0 neutron sub-library has increased significantly from those present in the ENDF/B-VII.1 release, many short-lived nuclides represented in the CASMO5 data library may not possess an evaluation. Therefore, the TALYS-based Evaluated Nuclear Data Library 2017 (TENDL-2017) [8,9] is used to supplement the ENDF/B-VIII.0 evaluation.

\section{UPDATED CASMO5 LIBRARY GENERATION PROCESS}

A depiction of the CASMO5 library generation procedure is shown in Fig. 1, which has been adapted from reference [10]. The ENDF/B-VIII.0 neutron cross section, thermal scattering (TSL), radioactive decay and fission yield sub-libraries are depicted near the top of Fig. 1. The neutron and TSL sub-libraries are processed through a sequence of NJOY2016 [11] modules: MODER, RECONR, BROADR, HEATR, PURR, THERMR, and GROUPR. The NJOY2016 sequence is repeated for each individual nuclide present in the CASMO5 library. Once neutron data for a nuclide has been processed with NJOY2016, a modified version of the NJOY94 POWR module is used to read the NJOY2016 output tape and generate an ASCII text file in the expected format. After all nuclides have been processed with NJOY2016 and NJOY94, a utility program (NJXMRG) is used to generate pre-mixed materials available in the CASMO5 library. The resulting ASCII files are concatenated by the NJXLIB utility into a single file, labeled as the ASCII Library in Fig. 1. The ASCII Library is structured into seven sub-files, each of which corresponds to certain type of data for all nuclides, e.g., all resonance data for resonant nuclides is grouped together into a single sub-file for efficiency. Finally, the ASCII Library is converted into a binary file using the CASLIB utility code. Since Transport-Corrected P0 (TCP0) cross sections are written by the CASLIB code into the final library, a dedicated infinite-medium $P_{N}$ calculation must be performed by CASMO5 using a preliminary library. The output TCP0 cross sections from this calculation are subsequently embedded into CASLIB and the final CASMO5 library is generated. 


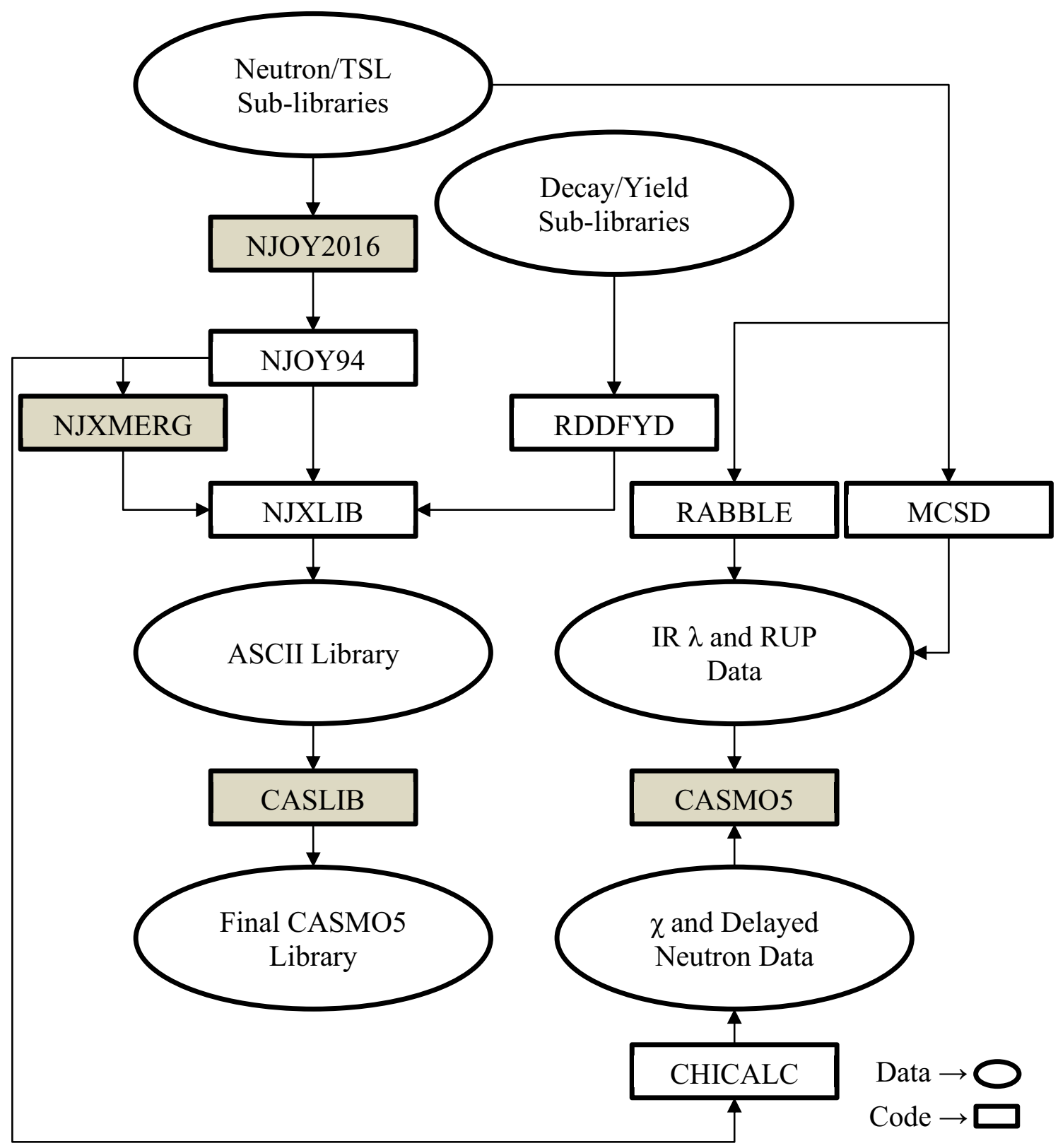

Figure 1. Updated CASMO5 library generation procedure. Codes highlighted in gray required updating to process ENDF/B-VIII.0 data.

The radioactive decay and fission yield sub-libraries are processed by the RDDFYD utility code. Either cumulative or independent fission yields can be used, which in turn depends on the depletion chains implemented in the CASMO5 code. The explicit representation (or omission) of metastable states of a certain nuclide in the CASMO5 depletion chains is also handled by RDDFYD. Finally, the determination of a representative incident-neutron energy is determined by RDDFYD when processing the fission yield data.

The generation of Intermediate Resonance (IR) $\lambda$ factors and Resonance Upscatter (RUP) corrections are performed by the RABBLE [12] and MCSD [13] codes, respectively. Delayed neutron data and fission spectra $\chi$ are extracted from the NJOY94 output via the CHICALC utility code. 
These data are not placed in the CASMO5 library and instead are embedded into the source code, as depicted in Fig. 1, which allows for greater versatility when testing options, such as RUP [7].

\section{NEW CASMO5 ENDF/B-VIII.0-BASED E8R0 LIBRARY}

Various advanced numerical schemes and features have been implemented into CASMO5 since the original release [1]. The CASMO5 neutron data library has also been updated from the original ENDF/BVII.1-based E7R1 200-series library [10] to the current E7R1 202-series library. The new E8R0 300series library shares the following features with the E7R1 202-series library:

- 586 energy groups with 128 fast groups $(20 \mathrm{MeV}$ to $9.118 \mathrm{keV}), 41$ resonance groups $(9.118 \mathrm{keV}$ to $10 \mathrm{eV}), 375$ fine groups $(10 \mathrm{eV}$ to $0.625 \mathrm{eV})$, and 42 thermal groups (below $0.625 \mathrm{eV}$ ).

- 1095 nuclides and materials with cross section data (full or absorption-only).

- Over 2300 nuclear reactions models including $(n, 2 n),(n, 3 n)$ and $(n, 4 n)$ reactions.

- A total of 119 heavy nuclides (from ${ }^{221} \mathrm{Rn}$ to ${ }^{255} \mathrm{Fm}$ ) and 491 fission products available in the library.

- Explicit transmutation chains for heavy nuclides (from ${ }^{229} \mathrm{Th}$ to ${ }^{252} \mathrm{Cf}$ ), fission products (from ${ }^{76} \mathrm{Ge}$ to ${ }^{165} \mathrm{Ho}$ ), burnable absorbers (AIC, Sm, Eu, Gd, Tb, Dy, Ho, Er, Hf, and W chains) and light nuclides $\left({ }^{1} \mathrm{H}\right.$ to ${ }^{20} \mathrm{O}$ unified chain).

- Shielded resonance data tabulated at 19 background cross sections and up to 10 temperatures ranging from $239 \mathrm{~K}$ to $2700 \mathrm{~K}$.

- High-order scattering matrices supporting 2D transport calculation with anisotropic sources.

- No ad hoc adjustment to ${ }^{238} \mathrm{U}$ resonance absorption as done for ENDF/B-VI data [10].

The new E8R0 300-series library features the following updated data from the ENDF/B-VIII.0 release:

- Absorption, fission, transport, and scattering (including $P_{N}$ ) cross sections.

- Radioactive decay and fission yield.

- Prompt and delayed neutron fission spectra, along with IR $\lambda$ factors and RUP correction.

- Energy release per fission and capture data.

\section{INITIAL VALIDATION OF NEW CASMO5 E8R0 LIBRARY}

A summary of the initial validation of the new CASMO5 E8R0 library is presented in this section. All CASMO5 calculations were performed using a 95 energy-group structure. Energy condensation from the 586-group library structure to the 95 groups is performed through a set of one-dimensional pin cell calculations. The 2D transport solution uses the default Linear Source (LS) Method of Characteristics (MOC) scheme and angular quadrature (64 azimuthal angles, 3 polar angles and a $0.05 \mathrm{~cm}$ ray spacing). All numerical results use the TCP0 unless otherwise noted. The problem-specific axial bucklings, used to model the axial leakage effects, have been gleaned from the various critical experiment reports.

\subsection{B\&W 1810 Critical Experiment Series}

The Babcock \& Wilcox 1810 critical experiments [14] represent realistic PWR reactor configurations. The core configurations consist of $5 \times 5$ arrays of Westinghouse, or Babcock \& Wilcox, $15 \times 15$ assemblies (Cores 1 through 17) and Combustion Engineering 16×16 assemblies (Cores 18 through 20), as depicted in Fig. 2. Cores 1 through 10 consist of uniform fuel enrichment, whereas Cores 12 through 17 consist of a high enriched central area surrounded by a low enriched zone. The central pseudo-assembly is modified by introducing gadolinium fuel pins, Ag-In-Cd (AIC) or $\mathrm{B}_{4} \mathrm{C}$ control rods, or even hollow tubes. Cores 18 through 20 also consist of a high enriched central area surrounded by a low enriched zone. These cores only differ in the number of gadolinium fuel pins present. All measurements are reported at a facility temperature of $25^{\circ} \mathrm{C}$. Tables I and II show the k-eff and Fission Rate (FR) results, respectively. 

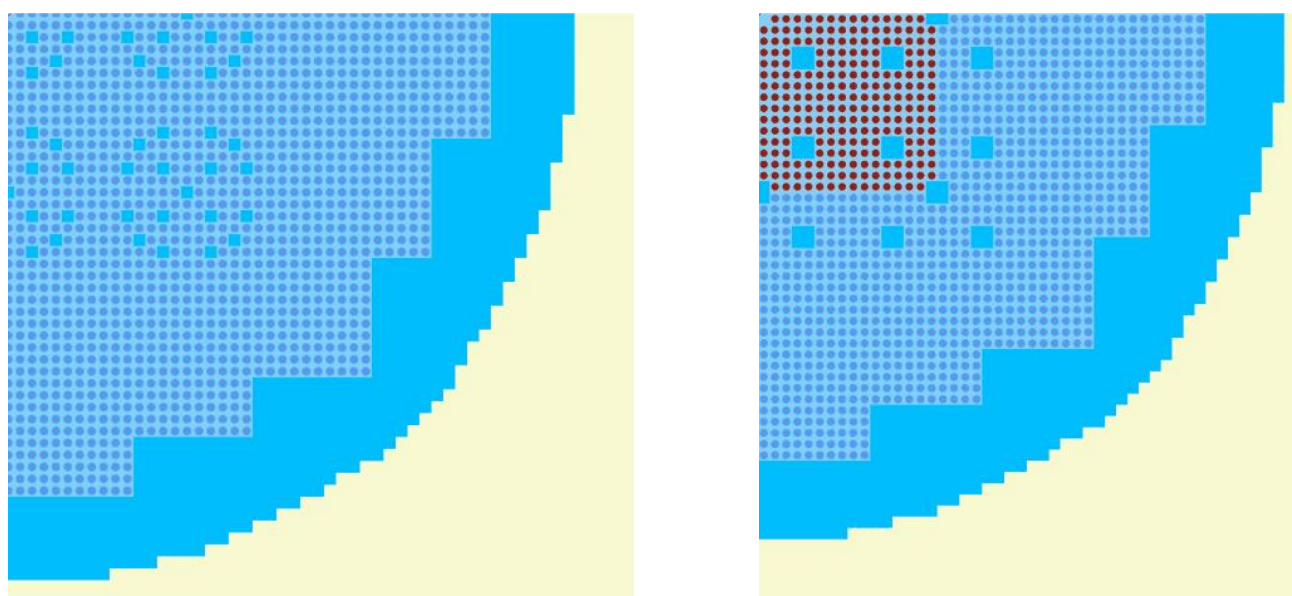

Figure 2. CASMO5 multi-assembly depiction of B\&W 1810 Core 1 (left) and Core 18 (right). Core 1 is similar to cores $2-17$ and Core 18 is similar to cores 19-20.

Table I. CASMO5 B\&W 1810 critical experiments: k-eff results.

\begin{tabular}{|c|c|c|c|c|c|c|}
\hline Core & Boron (ppm) & Lattice & Central Region & $4 \% \mathrm{Gd}^{1}$ Pins & AIC Rods & $k-e f f$ \\
\hline 1 & 1337.9 & $15 \times 15$ & Uniform & -- & -- & 1.00125 \\
\hline 2 & 1250 & $15 \times 15$ & Uniform & -- & 16 & 1.00088 \\
\hline 3 & 1239.3 & $15 \times 15$ & Uniform & 20 & -- & 1.00111 \\
\hline 4 & 1171.7 & $15 \times 15$ & Uniform & 20 & 16 & 1.00174 \\
\hline 5 & 1208 & $15 \times 15$ & Uniform & 28 & -- & 1.00073 \\
\hline $5 \mathrm{~A}$ & 1191.3 & $15 \times 15$ & Uniform & 32 & -- & 1.00075 \\
\hline $5 \mathrm{~B}$ & 1207.1 & $15 \times 15$ & Uniform & 28 & -- & 1.00087 \\
\hline 6 & 1155.8 & $15 \times 15$ & Uniform & 28 & 16 & 1.00102 \\
\hline $6 \mathrm{~A}$ & 1135.6 & $15 \times 15$ & Uniform & 32 & 16 & 1.00101 \\
\hline 7 & 1208.8 & $15 \times 15$ & Uniform & 28 (annular) & -- & 1.00083 \\
\hline 8 & 1170.7 & $15 \times 15$ & Uniform & 36 & -- & 1.00093 \\
\hline 9 & 1130.5 & $15 \times 15$ & Uniform & 36 & 16 & 1.00085 \\
\hline 10 & 1177.1 & $15 \times 15$ & Uniform & 36 & 16 & 1.00076 \\
\hline 12 & 1899.3 & $15 \times 15$ & 2-Region & -- & -- & 1.00162 \\
\hline 13 & 1635.4 & $15 \times 15$ & 2-Region & -- & 16 & 1.00204 \\
\hline 14 & 1653.8 & $15 \times 15$ & 2-Region & 28 & 16 & 1.00143 \\
\hline 15 & 1479.7 & $15 \times 15$ & 2-Region & 28 & 16 & 1.00194 \\
\hline 16 & 1579.4 & $15 \times 15$ & 2-Region & 36 & -- & 1.00153 \\
\hline 17 & 1432.1 & $15 \times 15$ & 2-Region & 36 & 16 & 1.00158 \\
\hline 18 & 1776.8 & $16 \times 16$ & 2-Region & -- & -- & 1.00221 \\
\hline 19 & 1628.3 & $16 \times 16$ & 2-Region & 16 & -- & 1.00209 \\
\hline \multirow[t]{3}{*}{20} & 1499 & $16 \times 16$ & 2-Region & 32 & -- & 1.00199 \\
\hline & & & & & Average & 1.00133 \\
\hline & & & & & Stand. Dev. (pcm) & 49.3 \\
\hline
\end{tabular}

\footnotetext{
$1 \% \mathrm{Gd}$ indicates the mass fraction of $\mathrm{Gd}$ relative to $\mathrm{Gd}_{2} \mathrm{O}_{3}$ in a mixture of $\mathrm{Gd}_{2} \mathrm{O}_{3}+\mathrm{UO}_{2}$ in percentage.
} 
Table II. CASMO5 B\&W 1810 critical experiments: FR results for Central Assembly (CA) and Diagonal Pins (DP).

\begin{tabular}{|c|c|c|c|c|}
\hline Core & $4 \%$ Gd Pins & AIC Rods & CA FR RMS & DP FR RMS \\
\hline 1 & -- & -- & 0.57 & \\
\hline 5 & 28 & -- & 0.74 & 2.26 \\
\hline 12 & -- & -- & 0.84 & \\
\hline 14 & 28 & 16 & 1.46 & 5.76 \\
\hline 18 & -- & -- & 0.84 & \\
\hline 20 & 32 & -- & 1.53 & 3.99 \\
\hline
\end{tabular}

The average CASMO5 calculated k-eff using the E8R0 library is $133 \mathrm{pcm}$ higher than unity over a wide range of burnable absorber types and loadings. The FR statistics indicate a Root-Mean-Square (RMS) difference of less than $2 \%$ relative to the Central Assembly and less than $6 \%$ relative to diagonal pins. The CASMO5 E8R0 predictions are comparable to those obtained with the E7R1 library.

\subsection{B\&W 1484 Critical Experiment Series}

The Babcock \& Wilcox 1484 critical experiments [15] consist of twenty-one configurations involving low and high leakage cores, as well as PWR fuel storage configurations. A depiction of the Core I through VI configuration is shown in Fig. 3 and Core X and XI in Fig. 4. k-eff results are given in Table III.
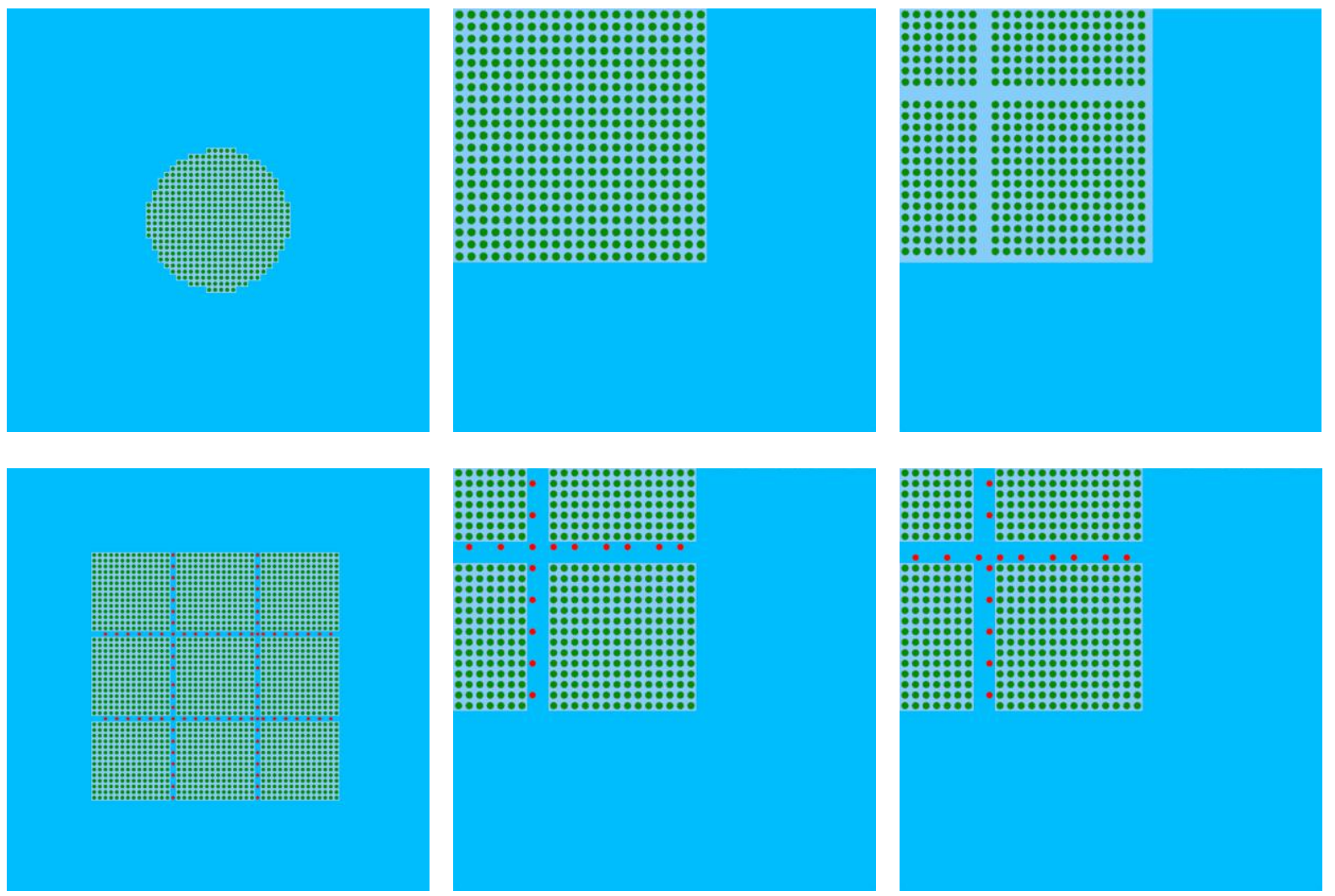

Figure 3. CASMO5 multi-assembly depiction of B\&W 1484 Cores I through VI (left to right, top to bottom). Absorber rods containing $\mathrm{B}_{4} \mathrm{C}$ are depicted as red pins. 

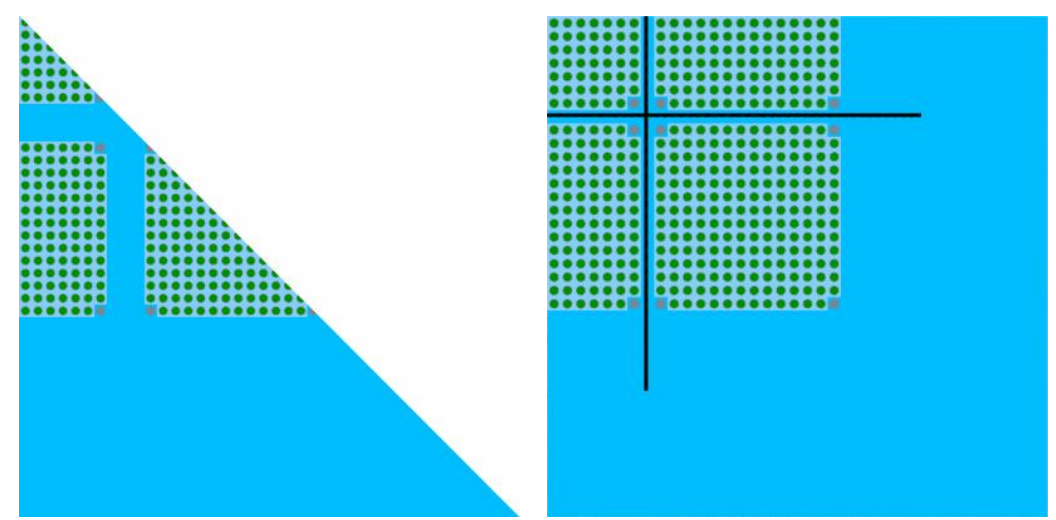

Figure 4. CASMO5 multi-assembly depiction of B\&W 1484 Core X (left) and XI (right). Isolation sheets are depicted as vertical and horizontal slabs.

Table III. CASMO5 B\&W 1484 critical experiments: k-eff results.

\begin{tabular}{|l|l|l|l|}
\hline Core & $\mathrm{k}$ & Core & $k-$ eff \\
\hline I & 1.00031 & X & 1.00270 \\
\hline II & 1.00071 & XIa & 1.00122 \\
\hline IIIa & 1.00025 & XIb & 1.00117 \\
\hline IIIb & 1.00063 & XIc & 1.00117 \\
\hline IIIc & 1.00022 & XId & 1.00123 \\
\hline IIId & 1.00021 & XIe & 1.00167 \\
\hline IIIe & 1.00033 & XIf & 1.00153 \\
\hline IIIf & 1.00062 & XIg & 1.00279 \\
\hline IIIg & 1.00059 & XII & 1.00082 \\
\hline IV & 0.99968 & XIII & 0.99906 \\
\hline V & 0.99887 & XIIIa & 0.99668 \\
\hline VI & 0.99903 & XIV & 0.99554 \\
\hline VII & 0.99958 & XV & 0.99182 \\
\hline VIII & 0.99965 & XVI & 0.99247 \\
\hline IX & 1.00028 & XVII & 0.99545 \\
\hline & & XVIII & 0.99558 \\
\hline & & XIX & 0.99715 \\
\hline & & XX & 0.99692 \\
\hline & & XXI & 0.99708 \\
\hline Mean & 1.00006 & Mean & 0.99853 \\
\hline St. dev. (pcm) & 56 & St. dev. (pcm) & 330 \\
\hline
\end{tabular}

Cores I and II do not involve any heterogeneity and only differ in size and shape. Core I consists of $2.459 \%$ enriched (by mass) ${ }^{235} \mathrm{U}$ fuel pins arranged in a high leakage circular core. Core II consists of the same fuel enrichment as Core I, but the core is arranged in a low-leakage configuration. Cores III through IX represent various hypothetical fuel storage configurations where $15 \times 15$ fuel assemblies are ranged in $3 \times 3$ space lattices. The spacing between the assemblies and interstitial absorber pin configuration is varied. Cores X through XXI also represent fuel storage configurations. Unlike the previous configurations, isolating/absorbing plates are introduced between the fuel assemblies. 
The CASMO5 average predicted k-eff for Cores I through IX is only $6 \mathrm{pcm}$ higher than unity and -147 pcm lower for Cores X through XXI. The second set of cores exhibits greater spatial non-uniformity with the presence of the absorber plates, where boron/aluminum isolation plates are present in Cores XIII through XXI. The CASMO5 E8R0 300 library predictions for the B\&W 1484 critical experiments are comparable to results obtained with the E7R1 202 library.

\subsection{DIMPLE S06A/B Critical Experiments}

The AEA Winfrith DIMPLE experimental program [16] conducted critical experiments involving a cruciform core configuration, which resembles a rectangular corner of a PWR core, and consists of five $16 \times 16$ PWR assemblies with $3 \%{ }^{235} \mathrm{U}$ enriched $\mathrm{UO}_{2}$ fuel pins. The DIMPLE S06A configuration is surrounded by a water reflector region, whereas the DIMPLE S06B configuration involves a $2.67 \mathrm{~cm}$ stainless steel baffle region between the fuel and water reflector regions. The DIMPLE critical experiments indicate the CASMO5 performance for multi-assembly calculations routinely performed to generate PWR reflector data. The geometry of critical experiments is depicted in Fig. 5.
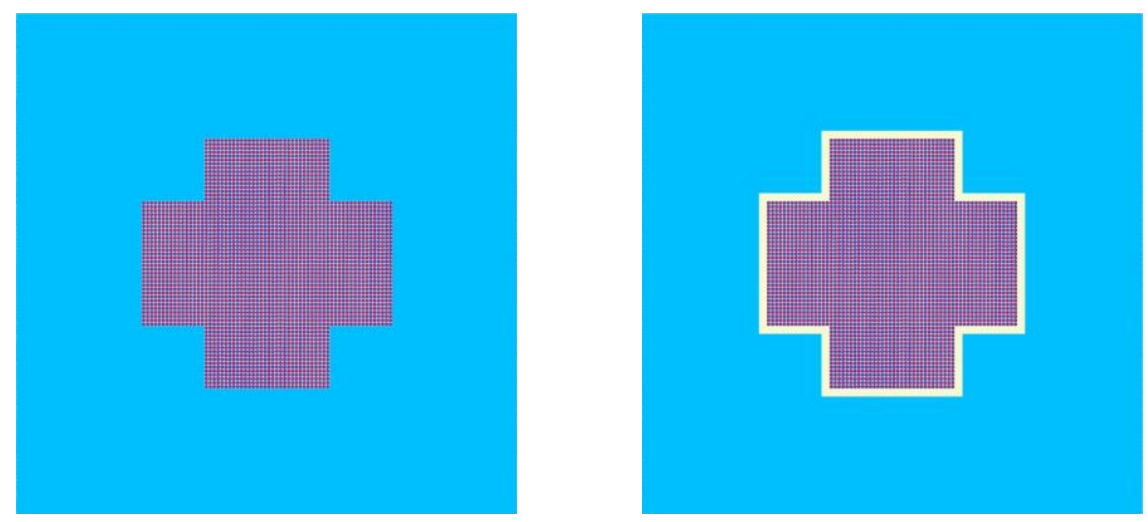

Figure 5. CASMO5 multi-assembly depiction of DIMPLE S06A (left) and S06B (right).

The k-eff for each core configuration are given in Table IV. Fission rates for ${ }^{235} \mathrm{U}$ and ${ }^{238} \mathrm{U}$ were also measured in select fuel pins. Table IV also provides descriptive statistics for the relative error in the CASMO5 fission rates compared to the measured values.

Table IV. CASMO5 DIMPLE critical experiments: k-eff and fission rate (relative difference in percent) results.

\begin{tabular}{|c|c|c|c|c|}
\hline & \multicolumn{2}{|c|}{ S06A } & \multicolumn{2}{c|}{ S06B } \\
\hline$k-e f f$ & \multicolumn{2}{|c|}{0.99888} & \multicolumn{2}{c|}{0.99909} \\
\hline Statistic & ${ }^{235} \mathrm{U}$ & ${ }^{238} \mathrm{U}$ & ${ }^{235} \mathrm{U}$ & ${ }^{238} \mathrm{U}$ \\
\hline Mean & 0.093 & 0.003 & -0.935 & -1.137 \\
\hline Standard Deviation & 0.779 & 2.24 & 1.43 & 1.904 \\
\hline RMS & 0.784 & 2.24 & 1.708 & 2.217 \\
\hline
\end{tabular}

The DIMPLE results indicate that the new CASMO5 E8R0 library performs well in predicting k-eff and fission rates for the generation of PWR reflector data involving baffle regions. Relative to the previous E7R1 202 library, the E8R0 library yields comparable results. 


\subsection{TCA Iron Reflector Critical Experiments}

The Tokai Research Establishment of JAERI conducted critical experiments using a Tank-type Critical Assembly (TCA) while varying the thickness of the steel or steel-water reflector slabs [17]. The fuel assembly consists of a $15 \times 15$ PWR design and $2.6 \%{ }^{235} \mathrm{U}$ enriched fuel. The objective of the experiment was to measure the reactivity effect of two reflector types: steel-only and steel-water reflector containing about $90 \%$ steel and $10 \%$ water. The TCA geometry for a steel-only reflector case is shown in Fig. 6 and a comparison of the CASMO5 predicted and measured reactivity effect [17] is shown in Fig. 7.

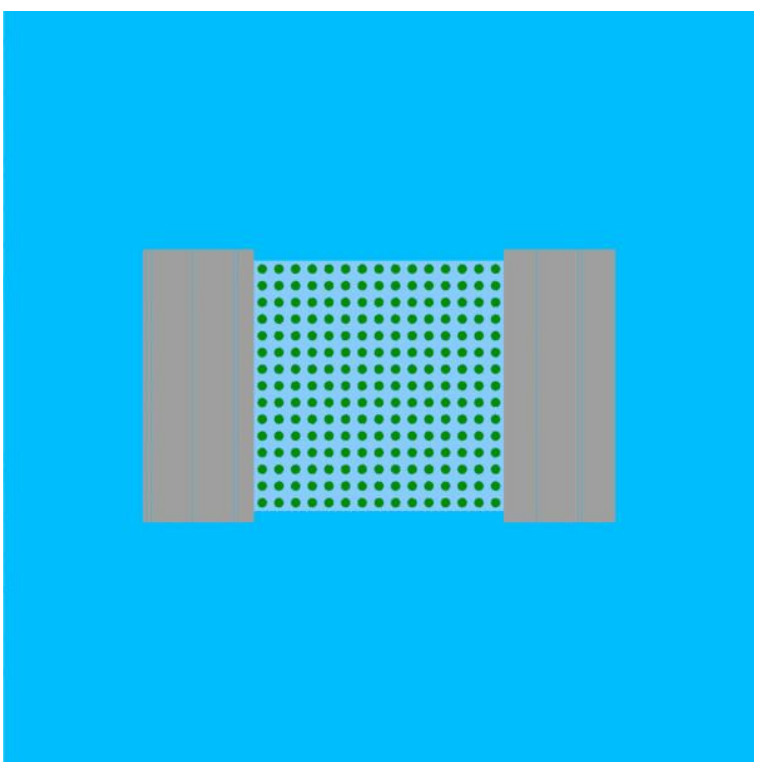

Figure 6. CASMO5 multi-assembly depiction of TCA steel-only reflector with $15.12 \mathrm{~cm}$ thickness.
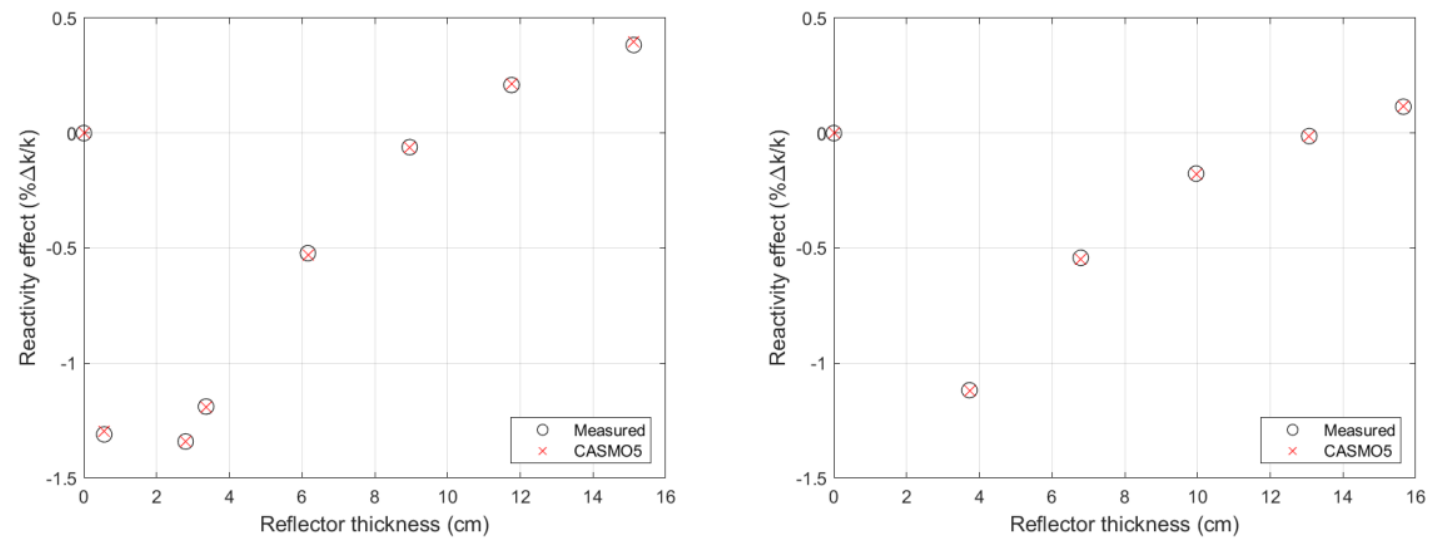

Figure 7. Reactivity effect of steel (left) and steel-water (right) reflectors in TCA experiment.

The reactivity effect is the change in reactivity from the reference critical water height, which is taken from the assembly with the steel replaced by water, to the critical height with the reflector present. The ratio of the effective delayed neutron yield and mean neutron lifetime, $\beta_{\text {eff }} / \Lambda$, is also provided for the bare TCA configuration. The CASMO5 E8R0 computed $\beta_{\text {eff }} / \Lambda$ is $162.8 \mathrm{~s}^{-1}$, as compared to a measured value of $161.5 s^{-1} \pm 5.0 s^{-1}$. The CASMO5 results using the E8R0 library are comparable to those obtained using the E7R1 202 library. 


\section{CONCLUSIONS}

A new commercially available CASMO5 nuclear data library has been generated based on the recentlyreleased ENDF/B-VIII.0 evaluation. A summary of the library generation procedure and main features of the new CASMO5 586-group library (referred to as E8R0) is provided in this work. Initial validation of the E8R0 library versus the B\&W 1810, B\&W 1484, DIMPLE and TCA critical experiments indicate comparable accuracy relative to the previous E7R1 202 library. Although changes were made to Fe cross sections in ENDF/B-VIII.0, they do not have a significant impact in terms of core $\mathrm{k}$-eff, such as in the TCA reflector experiments. Future work involves further validation against MOX critical experiments and comparisons to Post Irradiation Examination (PIE) isotopic data.

\section{REFERENCES}

1. J. Rhodes, K. Smith, and D. Lee, "CASMO-5 Development and Applications," Proc. Int. Conf. on Advances in Nuclear Analysis and Simulation (PHYSOR 2006), Vancouver, BC, Canada, Sept. 10-14 (2006).

2. T. Bahadir and S.-Ö. Lindhal, "Studsvik's Next Generation Nodal Code SIMULATE-5," Proc. Advances in Nuclear Fuel Management IV (ANFM IV), Hilton Head, SC, USA, April 12-15 (2009).

3. B. Haugh, "Generic Application of the Studsvik Scandpower Core Management System to Pressurized Water Reactors," SSP-14-P01/028-TR-NP-A, Studsvik Scandpower, Inc. (2017).

4. R. Ferrer, J. Hykes, and J. Rhodes, "Development of CASMO5 for VVER-1000/1200 Analysis and Preliminary Validation using Critical Experiments," Kerntechnik, 84, pp. 214-227 (2019).

5. T. Bahadir, "SIMULATE5-HEX Extension for VVER Analyses," Kerntechnik, 83, pp. 268-274 (2018).

6. D.A. Brown, et al., "ENDF/B-VIII.0: The $8^{\text {th }}$ Major Release of the Nuclear Reaction Data Library with CIELO-project Cross Sections, New Standards and Thermal Scattering Data," Nuclear Data Sheets, 148, pp. 1-142 (2018).

7. G. Grandi and R. Ferrer, "Effects of CASMO5 Models and Delayed Neutron Data on SIMULATE-3K Reactivity Initiated Accident Predictions," Proc. Int. Conf. on Reactor Physics paving the way towards more efficient systems (PHYSOR 2018), Cancun, Mexico, April 22-26 (2018).

8. A.J. Koning and D. Rochman, "Modern Nuclear Data Evaluation with the TALYS Code System," Nuclear Data Sheets, 113, pp. 2841-2934 (2012).

9. A.J. Koning, et al., "TENDL: Complete Nuclear Data Library for Innovative Nuclear Science and Technology," Nuclear Data Sheets, 155, pp. 1-55 (2019).

10. J. Rhodes, N. Gheorghiu, and R. Ferrer, "CASMO5 JENDL-4.0 and ENDF/B-VII.1beta4 Libraries," Proc. Int. Conf. on Advances in Reactor Physics - Linking Research, Industry, and Education (PHYSOR 2012), Knoxville, TN, USA, April 15-20 (2012).

11. R.E. MacFarlane, et al., "The NJOY Nuclear Data Processing System, Version 2016," LA-UR-1720093, Los Alamos National Laboratory, Los Alamos, NM (2018).

12. U. Decher, "Users Manual for RABBLE, DIT Cross Section Library Preparation," Nuclear Data Project Report NDP-032 Rev. 1, ABB Combustion Engineering Nuclear Fuel (1994).

13. D. Lee, K. Smith, and J. Rhodes, "The Impact of ${ }^{238}$ U Resonance Elastic Scattering Approximations on Thermal Doppler Reactivity," Ann. Nucl. Energy, 36, pp. 274-280 (2009).

14. L.W. Newman, "Urania-Gadolinia: Nuclear Model Development and Critical Experiment Benchmark,” DOE/ET/34212-41 BAW-1810, Babcock \& Wilcox, Lynchburg, VA (1984).

15. M.N. Baldwin, et al., "Critical Experiments Supporting Close Proximity Water Storage of Power Reactor Fuel,” BAW-1484-7, Babcock \& Wilcox, Lynchburg, VA (1979).

16. D. Hanlon and B. Franklin, "Light Water Moderated and Reflected Low Enriched Uranium (3 wt. \% U-235) Dioxide Rod Lattices DIMPLE S06,” DIMPLE-LWR-EXP-002, IRPhEP, (2006).

17. Y. Tahara, H. Sekimoto, and Y. Miyoshi, "Reactivity Effect of Iron Reflector in LWR Cores," Journal of Nuclear Science and Technology, 38 (2), pp. 102-111 (2001). 